\title{
NONLINEAR AND LINEAR TIMESCALES NEAR KINETIC SCALES IN SOLAR WIND TURBULENCE
}

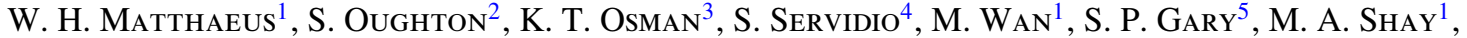 \\ F. Valentini ${ }^{4}$, V. Roytershteyn ${ }^{6}$, H. Karimabadi ${ }^{6}$, and S. C. Chapman ${ }^{3,7}$ \\ ${ }^{1}$ Department of Physics and Astronomy, University of Delaware, DE 19716, USA; whm@udel.edu \\ 2 Department of Mathematics, University of Waikato, Hamilton, New Zealand \\ ${ }^{3}$ Centre for Fusion, Space, and Astrophysics, University of Warwick, Coventry CV4 7AL, UK \\ ${ }^{4}$ Dipartimento di Fisica, Università della Calabria, I-87036 Cosenza, Italy \\ 5 Space Sciences Institute, Boulder, CO 80301, USA \\ ${ }^{6}$ Sciberquest, Inc., Del Mar, CA 92014, USA \\ ${ }^{7}$ Max Planck Institute for the Physics of Complex Systems, D-01187 Dresden, Germany \\ Received 2014 April 22; accepted 2014 June 15; published 2014 July 17
}

\begin{abstract}
The application of linear kinetic treatments to plasma waves, damping, and instability requires favorable inequalities between the associated linear timescales and timescales for nonlinear (e.g., turbulence) evolution. In the solar wind these two types of timescales may be directly compared using standard Kolmogorov-style analysis and observational data. The estimated local (in scale) nonlinear magnetohydrodynamic cascade times, evaluated as relevant kinetic scales are approached, remain slower than the cyclotron period, but comparable to or faster than the typical timescales of instabilities, anisotropic waves, and wave damping. The variation with length scale of the turbulence timescales is supported by observations and simulations. On this basis the use of linear theory-which assumes constant parameters to calculate the associated kinetic rates - may be questioned. It is suggested that the product of proton gyrofrequency and nonlinear time at the ion gyroscales provides a simple measure of turbulence influence on proton kinetic behavior.
\end{abstract}

Key words: magnetohydrodynamics (MHD) - solar wind - Sun: corona - turbulence

Online-only material: color figures

\section{INTRODUCTION}

Plasma physics often employs simplified frameworks to explain properties of observed plasmas in solar, space, and astrophysics. Prominent among these is the large class of calculations based upon linearization of a Vlasov description about a uniform equilibrium magnetized state. A rich variety of normal modes and wave damping rates emerge, even when each plasma species possesses simple properties such as Maxwellian distributions with isotropic temperatures. The dependence of these modes on species plasma betas and other dimensionless parameters is a familiar and important topic in space plasma physics and astrophysics. More complex distribution functions that admit temperature anisotropy or beams are familiar in low collisionality solar wind, accretion disks, and galaxy clusters (e.g., Sharma et al. 2007; Schekochihin et al. 2010; Kunz et al. 2011; Riquelme et al. 2012). These features provide free energy for families of instabilities such as the firehose, Alfvén ion cyclotron, and mirror mode instabilities (e.g., Gary 1993). The characteristic timescales (or reciprocal frequencies) of the relevant linear Vlasov modes typically extend over a very wide range.

Intriguingly, many plasmas of interest-including the solar wind, corona, and interstellar medium-also exhibit properties of a turbulence cascade extending from larger magnetohydrodynamic (MHD) to smaller kinetic spatial scales. Cascades also can extend over a wide range of timescales. The present paper compares linear Vlasov timescales and nonlinear turbulence timescales as the associated length scales approach the transition between MHD and kinetic regimes. We have in mind the specific case of the solar wind, for which it is possible to inform the discussion using analytical estimates, simulations, and direct observational analysis. We will conclude that nonlinear and linear inverse timescales can be comparable, with frequencies of order of $1 / 100$ to $1 / 10$ the proton gyrofrequency, for the oblique wave vectors thought to dominate solar wind fluctuations. Therefore caution is required in applying the static equilibrium assumptions underlying much of linear theory. Finally we close with a suggestion for a simple dimensionless measure of the degree of turbulence cascade effects on kinetic processes. Note that throughout the presentation we avoid committing to a specific dynamical model of the spectrum, such as reduced MHD, two-dimensional MHD, or Goldreich-Sridhar theory (Montgomery 1982; Shebalin et al. 1983; Oughton et al. 1994; Goldreich \& Sridhar 1995; Zhou et al. 2004), in order to maintain as broad a context as possible; however, some issues related to anisotropic spectral models are discussed in Appendix A.

\section{TIMESCALES IN LINEAR VLASOV PLASMA}

From a technical perspective, linearization leads to small amplitude solutions having exponential behavior $\exp \left(i \omega_{c} t\right)$ with complex frequencies $\omega_{c}=\omega+i \gamma$ consisting of real frequency $\omega$ and a growth $(\gamma>0)$ or damping rate $(\gamma<0)$. In general, $\omega$ and $\gamma$ are functions of the wave vector $\boldsymbol{k}$, not just its magnitude $k=|\boldsymbol{k}|$. Linearization about a uniform state yields normal modes of the plasma. These are generally transient (Barnes 1979), but some have small damping rates $\gamma<0$ with $|\gamma| /|\omega| \ll 1$. Besides damped waves, relevant instabilities are studied in linear Vlasov calculations by perturbing about a simple plasma configuration (e.g., Gary 1993). A typical unstable equilibrium might have uniform density and magnetic field $\boldsymbol{B}_{0}$, with free energy supplied by an anisotropic particle distribution function.

To discuss a range of relevant timescales for normal modes, it is convenient to adopt a normalization that expresses timescales in units of the proton gyrofrequency $\Omega_{\mathrm{ci}}=e B / m c$, in terms 
of a characteristic magnetic field strength $B$, and length in units of the ion inertial scale $d_{\mathrm{i}}=c / \omega_{\mathrm{pi}} \equiv V_{A} / \Omega_{\mathrm{ci}}$, where $\omega_{\mathrm{pi}}$ is the plasma frequency, $c$ the light speed, $\rho$ the mass density, and $V_{A}=B / \sqrt{4 \pi \rho}$ the Alfvén speed. Kinetic scales will be indicated when $k d_{\mathrm{i}} \sim 1$ or greater, while short timescales, $\tau(\boldsymbol{k})$, are indicated by $\Omega_{\mathrm{ci}} \tau(\boldsymbol{k}) \sim 1$ or less. The actual values of the frequencies associated with waves and instabilities are obtained either by numerical solutions of the full dispersion equation (Gary 1993; Lysak \& Lotko 1996) or through analytic approximations (Hollweg 1999).

When the relatively low-frequency MHD waves-Alfvén and fast and slow magnetosonic waves - are extended to a kinetic description, one finds in linear Vlasov theory that the magnetosonic waves are much more heavily damped (Barnes 1966, 1968, 1969) than the Alfvén mode for relevant parameters. This damping is often invoked as a basic physical explanation for frequently observed fluctuations that resemble the Alfvén waves in the inner heliosphere (Belcher \& Davis 1971; Roberts et al. 1987).

Considerable effort has been devoted to describing normal modes that may be present in the kinetic range of solar wind turbulence, where there is a well-known observed dominance of quasi-two-dimensional wave vectors (Matthaeus et al. 1990; Leamon et al. 2000; Osman \& Horbury 2007); that is, perpendicular wave number $k_{\perp}=\left|\boldsymbol{k} \times \hat{\boldsymbol{B}}_{0}\right| \gg$ parallel wave number $k_{\|}=\left|\boldsymbol{k} \cdot \hat{\boldsymbol{B}}_{0}\right|$. In the following sections we therefore emphasize discussion of the properties of oblique fluctuations that are likely to make up a substantial fraction of the solar wind fluctuation spectrum.

Within this class a popular choice is the oblique kinetic Alfvén wave (KAW; Hollweg 1999; Leamon et al. 1999) with wave frequencies $\omega<\Omega_{\mathrm{ci}}$ low compared to the proton cyclotron frequency $\Omega_{\text {ci }}$. Recent observations also suggest that such lowfrequency modes are energetically most relevant in the solar wind between the ion and electron inertial scales (Bale et al. 2005; Howes et al. 2006; Alexandrova et al. 2009; Sahraoui et al. 2010). For this reason we will focus here on wave properties approaching and near $k d_{\mathrm{i}}=1$, and on wave vectors mainly in the oblique and quasi-two-dimensional range of angles to the mean field, that is $60^{\circ}<\theta<90^{\circ}$. Figures 1,3 , and 4 portray this emphasis on oblique wave vectors by progressively shading the linear results in the more oblique range of angles.

Higher frequency waves may also be present, such as whistlers (Chang et al. 2013) or Alfvén ion cyclotron (AIC) waves with quasi-parallel wave vectors, although these are generally thought to occur at a relatively lower amplitude and higher frequency. AIC modes are particularly relevant in models involving pitch angle scattering (Isenberg \& Vasquez 2011).

The observed frequencies and wave vectors of fluctuations near ion kinetic scales have been analyzed in terms of linear wave theory (Sahraoui et al. 2010, 2012; Roberts et al. 2013). Due to the ambiguities inherent in these analyses, the main conclusion that can be drawn is that the observed fluctuations in about a decade of scale near $k d_{\mathrm{i}} \sim 1$ are "low-frequency" and are consistent with a dominant contribution of kinetic Alfvén waves with wave vectors lying in the range of $85^{\circ}-89^{\circ}$ of the mean magnetic field. Interpreted as waves, such fluctuations have frequencies roughly in the range $\omega / \Omega_{\mathrm{ci}} \sim 10^{-1}$ to $10^{-2}$. Indeed, linear Vlasov theory (see Figure 1) shows that the frequencies of highly oblique KAWs are very low for wavelengths near the ion inertial scale. At relatively short wavelengths $\left(k d_{\mathrm{i}} \gg 1\right)$ and relatively high frequencies $\left(\omega / \Omega_{\mathrm{ci}} \gg 1\right)$, whistler waves can propagate with relatively weak damping at directions both
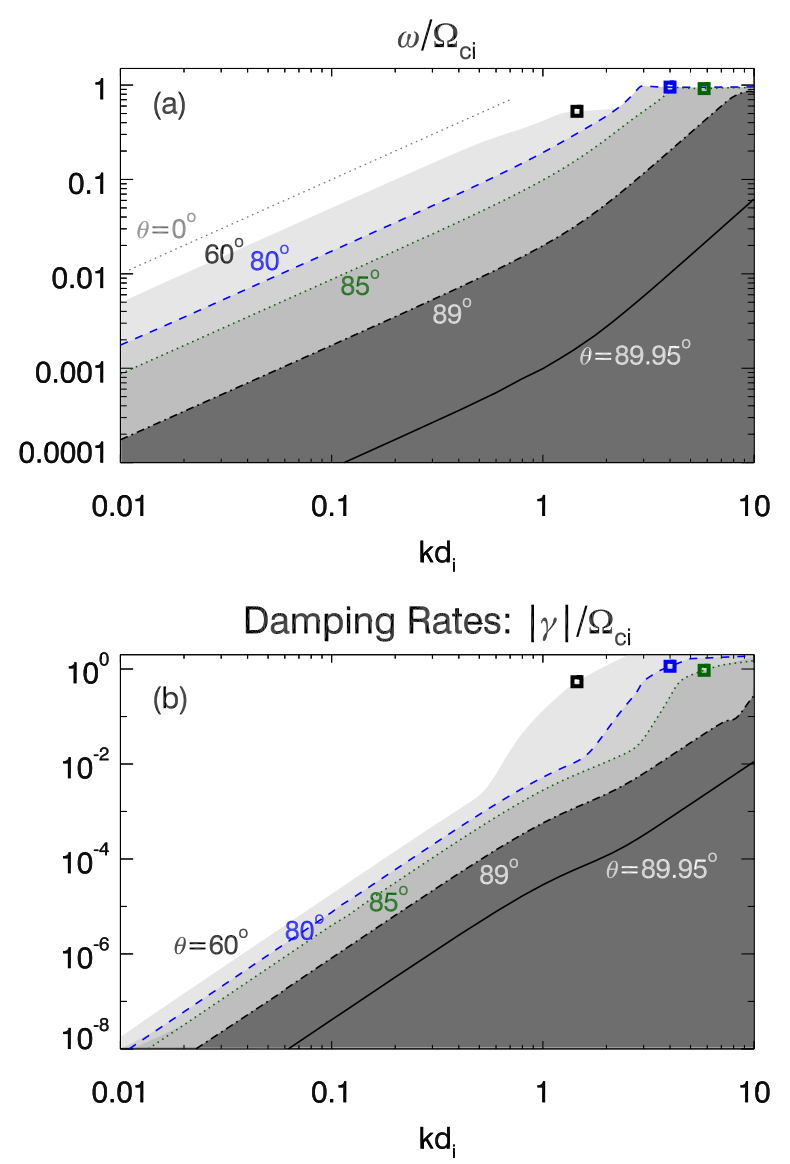

Figure 1. Illustration of the range of wave frequencies obtained from linear Vlasov theory using typical solar wind parameters, emphasizing oblique and quasi-two-dimensional wave vectors with angles to the mean magnetic field in the range $60^{\circ}<\theta<90^{\circ}$, which are commonly thought to be most relevant for solar wind turbulence cascade. More oblique regions are shaded in a darker hue. (a) Real frequencies of Alfvén waves and KAWs. The curves at specific $\theta$ are for a $\beta_{\mathrm{p}}=1$ plasma. (b) Wave damping rates, $|\gamma|$, for the same cases as in panel (a). Note that in the KAW regions there are cases where $|\gamma(\boldsymbol{k})|>\omega(\boldsymbol{k})$ and thus damping is too strong for waves to be properly excited. The first value of $k d_{\mathrm{i}}$ where this occurs, at each $\theta$, is indicated by the square symbol.

(A color version of this figure is available in the online journal.)

parallel and oblique to the background magnetic field (e.g., Gary et al. 2008). However, there is substantial current debate as to whether such modes make a significant contribution to the short-wavelength turbulent spectra observed in the solar wind.

The damping rates of normal modes such as KAWs are also relevant, for example, in some theories of solar wind heating that invoke a balance between linear damping and cascade rate (Barnes 1969; Leamon et al. 1999; Howes et al. 2008). Linear damping rates have been computed and tabulated for both nearly parallel wave vectors (Gary \& Borovsky 2004) as well as highly oblique orientations (Leamon et al. 1999; Sahraoui et al. 2010). Damping rates for modes with wave number close to ion kinetic scales $k d_{\mathrm{i}} \sim 1$ (within a decade or so) are frequently found to be of order $\gamma / \Omega_{\mathrm{ci}} \sim 10^{-1}$ to $10^{-2}$ (Lysak \& Lotko 1996; Leamon et al. 1999; Gary \& Borovsky 2004). This characteristic range of damping rates is found for a reasonably wide range of electron plasma beta and for ratios of ion to electron temperatures from zero to 10 (Lysak \& Lotko 1996). Depending upon parameters and angle of the wave vector to the mean magnetic field, this damping rate may vary considerably. Figure 1(b) displays damping rates for Alfvén waves and KAWs in an electron-proton plasma with proton plasma beta $\beta_{\mathrm{p}}=1$. 

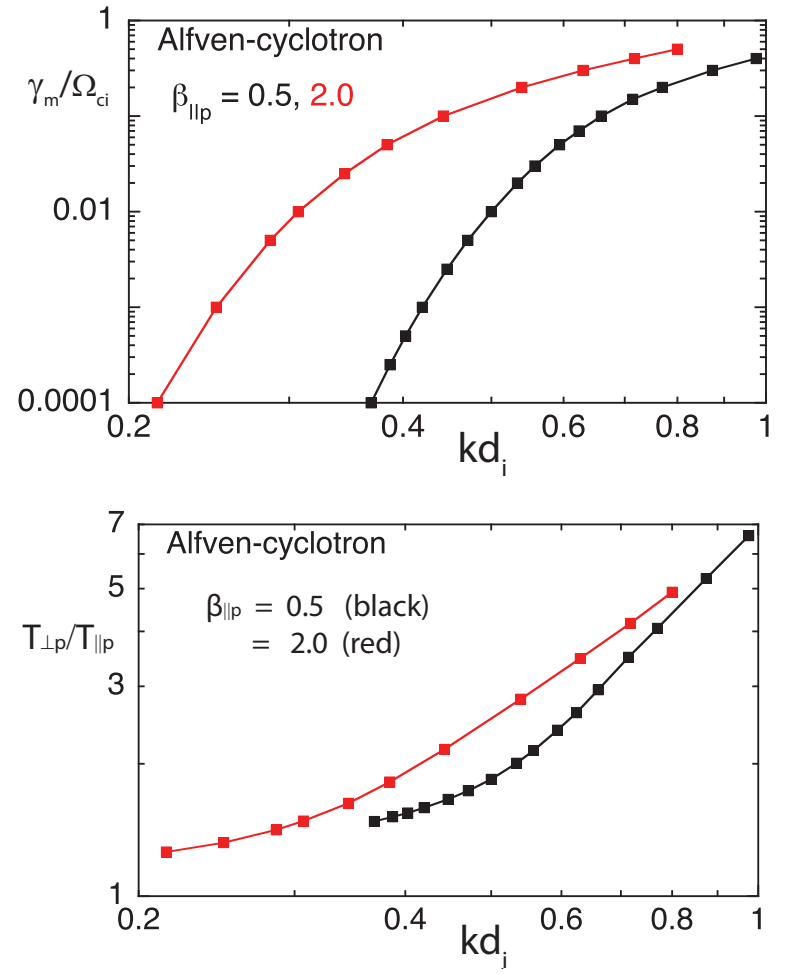

Figure 2. (a) Maximum growth rates $\gamma_{m}$ normalized to ion cyclotron frequency $\Omega_{\mathrm{ci}}$ vs. wave number normalized to ion inertial length $d_{\mathrm{i}}$, for the Alfvén cyclotron instability, for two values of $\beta_{\| \mathrm{p}}$ typical of the solar wind. (b) Corresponding proton temperature anisotropies for the cases shown in panel (a). Note that plasma with values of $\beta_{\|}$and $T_{\perp p} / T_{\| p}$ such that the growth rate $>0.05 \Omega_{\mathrm{ci}}$ are rarely observed in the solar wind.

(A color version of this figure is available in the online journal.)

Note that for $k d_{\mathrm{i}} \gtrsim 1$, putative KAWs at oblique angles have $|\gamma(\boldsymbol{k})| / \omega(\boldsymbol{k})>1$ and thus are so strongly damped that it is difficult to excite them.

If the ion and electron velocity distributions are nearly thermal, that is, approximately Maxwellian, kinetic linear dispersion theory predicts that the normal modes of the plasma are undamped or weakly damped. However, if a species velocity distribution is sufficiently anisotropic, dispersion theory as well as kinetic simulations show that normal modes grow in time, leading to instability. Various anisotropies drive a variety of instabilities [Gary, 1993]; a source of free energy often observed in the solar wind is the $T_{\perp \mathrm{p}} / T_{\| \mathrm{p}}>1$ proton temperature anisotropy.

Under typical solar wind conditions, there are two distinct growing modes which arise as a result of this anisotropy: the proton mirror instability and the Alfvén-cyclotron instability. The former has zero real frequency in a homogeneous plasma, has maximum growth rate $\gamma_{\mathrm{m}}$ at relatively oblique propagation $\left(0<k_{\|}<k_{\perp}\right)$, and is favored at relatively high values of $\beta_{\| \mathrm{p}}$. The latter mode satisfies $0<\omega<\Omega_{\mathrm{ci}}$, has maximum growth rate at $\boldsymbol{k} \times \boldsymbol{B}_{0}=\mathbf{0}$, and is preferentially excited if $\beta_{\| \mathrm{p}}<1$. As the proton temperature anisotropy is increased, Figure 2(a)-(b), illustrating the typical solar wind parameter range of $0.5<\beta_{\| \mathrm{p}}<2.0$, show that linear dispersion theory predicts that both $\gamma_{\mathrm{m}}$ and the corresponding wave number $k d_{\mathrm{i}}$ also increase in magnitude. Spacecraft observations (Hellinger et al. 2006; Matteini et al. 2007; Bale et al. 2009; Maruca et al. 2011) show that scattering by enhanced fluctuations from instabilities acts to constrain proton anisotropies. The typical extremal anisotropy values correspond to relatively weak growth rates, that is, $10^{-3}<\gamma_{\mathrm{m}} / \Omega_{\mathrm{ci}} \lesssim 0.05$. Figure 2(a) shows that this growth rate corresponds to $0.25<k d_{\mathrm{i}}<0.6$. This implies that the fluctuations that may be produced by protondriven instabilities would be expected to have their maximum amplitudes at wavelengths near (or slightly larger than) the ion inertial scale, which typically marks the end of the inertial range spectrum, as discussed below.

Summarizing these linear theory results, near the scales associated with onset of the kinetic physics range $\left(k d_{i} \sim 1\right)$ there are highly relevant waves with frequencies on the order of a few tenths of the cyclotron frequency or less, and associated damping rates of similar magnitude that may contribute to dissipation. Likewise, temperature anisotropy-driven instabilities at limiting parameters in the solar wind are reported to have typical growth rates that are also less than a tenth of the cyclotron frequency.

\section{NONLINEAR CASCADE TIMESCALES}

Plasma dynamics at finite amplitudes permit nonlinear couplings that are contemporaneous with linear processes. Sufficiently strong nonlinearity drives a cascade that potentially influences dynamics across decades of scale. In the solar wind this picture is supported by observation of power-law energy spectra, evolving Alfvén ratio and cross helicity, and the distribution of plasma heating (e.g., Dobrowolny et al. 1980; Goldstein et al. 1995; Tu \& Marsch 1995; Matthaeus \& Velli 2011). The cascade is also evidenced directly, by observation of third-order statistics (Sorriso-Valvo et al. 2007; Marino et al. 2008; MacBride et al. 2008).

Accompanying broadband spatial structure, a wide range of timescales also characterizes the cascade. ${ }^{8}$ These nonlinear timescales generally become smaller at smaller scales. This speed-up is important as it is responsible for the tendency of turbulence to attain quasi-universal small-scale statistical equilibria (Batchelor 1970). The question at hand is whether, as the kinetic plasma range is approached in scale (and at appropriate oblique angles to the mean magnetic field), the nonlinear timescales are competitive with timescales emerging from the linear processes summarized in the previous section. This comparison relates to the balance between cascade activity-mainly mediated by the nonlinear time (see below, and Appendix A) — and linear kinetic effects that occur independently at each wave vector without regard for cross-scale couplings.

For context, let us review the standard Kolmogorov phenomenology for steady-state isotropic hydrodynamics (e.g., Frisch 1995), focusing on the scale-dependence of the nonlinear timescales. We denote the (rms) turbulence amplitude as $u$ and the outer (or energy-containing) scale as $L$. The global cascade rate $\epsilon$ and the scale-dependent version $\epsilon_{\ell}$ are constrained as

$$
\epsilon \sim \frac{u^{3}}{L} \sim \epsilon_{\ell} \sim \frac{u_{\ell}^{3}}{\ell} \sim \frac{u_{\ell}^{2}}{\tau_{\ell}},
$$

in terms of inertial range scale $\ell$ and longitudinal velocity increment $u_{\ell}$. The last relation serves to define the scaledependent nonlinear time

$$
\tau_{\ell}=\frac{\ell}{u_{\ell}} \sim \frac{\ell^{2 / 3}}{\epsilon^{1 / 3}} .
$$

\footnotetext{
8 It is important to distinguish Eulerian timescales and Lagrangian timescales. The former may include fast timescales associated with sweeping and unidirectional wave propagation, which do not induce spectral cascade (Chen \& Kraichnan 1989; Servidio et al. 2011).
} 
The equivalent development in Fourier wave number $k$ characterizes a steady cascade, local in wave number, as

$$
\epsilon \sim \epsilon_{k} \sim \frac{u_{k}^{2}}{\tau_{\mathrm{nl}}(k)} .
$$

Here the amplitude of fluctuations at scale $1 / k$ is $u_{k}=\sqrt{k \mathcal{E}(k)}$ [dropping $O(1)$ factors] and the nonlinear time at wave number $k$ is

$$
\tau_{\mathrm{nl}}(k)=\frac{1}{k u_{k}} \sim \frac{L}{u} \frac{1}{(k L)^{2 / 3}} .
$$

In writing Equation (4) use is made of the steady Kolmogorov omnidirectional spectrum $\mathcal{E}(k) \sim \epsilon^{2 / 3} k^{-5 / 3}$ as defined in Batchelor (1970).

Extension of this result to MHD is straightforward (e.g., Zhou et al. 2004). For nearly incompressible MHD the relevant cascaded quantity is the total energy per unit mass, essentially $Z^{2}=u^{2}+b^{2}$. Here, $u$ and $b$ are the rms fluctuations in velocity and magnetic field, the latter measured in Alfvén speed units. For the present illustration we consider the simplest case in which, for the inertial range of scales, the cross helicity is near zero (i.e., uncorrelated $\boldsymbol{u}$ and $\boldsymbol{b}$ ), and $u^{2}$ and $b^{2}$ are of the same order. ${ }^{9}$ Then the above arguments are readily reformulated in terms of the total energy and $Z_{k}=\sqrt{k \mathcal{E}(k)}$, the amplitude near wave number $k$. Here, for incompressible MHD, the omnidirectional energy spectrum $\mathcal{E}(k)=\mathcal{E}^{u}(k)+\mathcal{E}^{b}(k)$ is the sum of the omnidirectional spectra for flow kinetic energy $\mathcal{E}^{u}(k)$ and fluctuation magnetic energy $\mathcal{E}^{b}(\mathrm{k})$. The relevant nonlinear timescale for a Kolmogorov analysis of MHD becomes

$$
\tau_{\mathrm{nl}}(k)=\frac{1}{k Z_{k}} \sim \frac{L}{Z} \frac{1}{(k L)^{2 / 3}},
$$

where in the latter relation, the modal amplitude $Z_{k}$ is written in terms of the global amplitude $Z$ using Kolmogorov scaling, in direct analogy to Equation (4).

It is also straightforward to introduce modifications in the above reasoning to treat the anisotropic perpendicular MHD cascade that is obtained in the presence of a strong imposed mean magnetic field (Shebalin et al. 1983; Zhou et al. 2004). For an assumed perpendicular cascade, the familiar procedure (e.g., Goldreich \& Sridhar 1995) is to simply interpret the scale $\ell$ and the wave number $k$ as the projection onto the perpendicular plane. Another potentially important timescale in describing the cascade is the Alfvén crossing time. However, for reasons outlined in Appendix A, we will base the following discussion of cascade timescales only on the nonlinear timescale.

Moving into the realm of kinetic plasma dynamics, there is no generally accepted formulation of a nonlinear timescale, in contrast to the fluid regime. However, we expect the same kind of hydrodynamic advective and line-stretching nonlinearities to be present in kinetic plasma. For $k d_{\mathrm{i}} \geqslant 1$ there should also be effects of the Hall current and other contributions to the generalized Ohm's law, which would change the estimate of the nonlinear timescale from $\tau_{\mathrm{nl}}(k) \sim 1 /\left(k Z_{k}\right)$ to something with a stronger dependence on $k$, such as $\tau_{\mathrm{nl}}(k) \rightarrow 1 /\left(d_{\mathrm{i}} k^{2} Z_{k}\right)$. The introduction of new timescales leads to the possibility of several different spectral scalings, an effect familiar in Hall MHD or electron MHD turbulence studies (e.g., Biskamp et al. 1999; Galtier \& Buchlin 2007; Alexandrova et al. 2008). Due to these

\footnotetext{
9 For the finite cross helicity case, see, e.g., Dobrowolny et al. (1980); Zhou et al. (2004); Matthaeus et al. (2004).
}

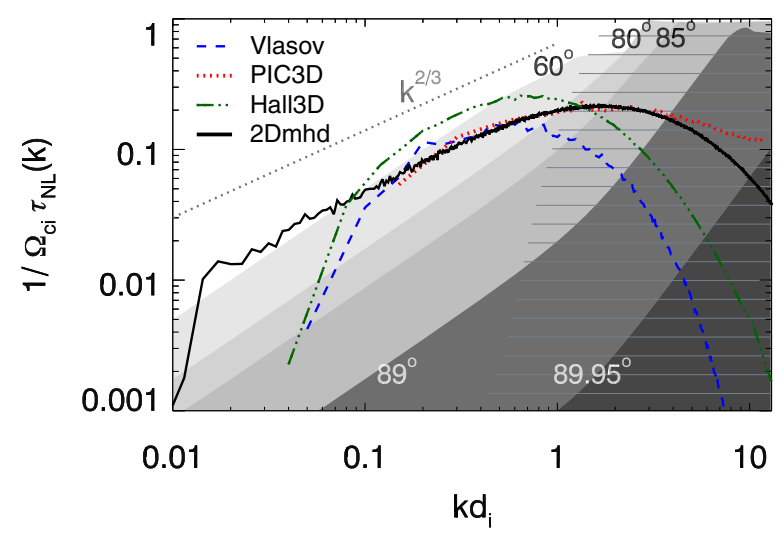

Figure 3. Wave number dependence of the estimated nonlinear rates computed from Equation (5) for several types of simulations (see text). As discussed in the text, no attempt is made to strictly control the simulation parameters. Nevertheless there is substantial physical similarity - the nonlinear rates increase as the scales decrease toward the dissipative or kinetic regime, and become comparable in magnitude to a tenth or more of the cyclotron frequency. Also shown is a reference $k^{2 / 3}$ line, associated with the scaling of $1 / \tau_{\mathrm{nl}}(k)$ for a steady Kolmogorov cascade. Shading corresponds to ranges of linear wave frequencies $\omega$, at increasingly oblique angles (darker shading). Horizontal hatching indicates corresponding linear theory damping rates $\gamma$ (cf. Figure 1).

(A color version of this figure is available in the online journal.)

complications (as well as the practical matter of the lack of high-frequency velocity data), in the analysis below we will restrict estimates to the simplest local-in-scale MHD nonlinear timescale. We expect that in the deep kinetic regime the MHD timescales will be upper bounds for the actual nonlinear timescales. However, we will defer to a future study a more careful and detailed treatment of the $k d_{i}>1$ nonlinear timescales.

With these caveats in mind, in all cases below we will estimate relevant nonlinear timescales by adopting the formulation given in Equation (5). The expected speed-up of the nonlinear rate is seen explicitly in the variation of $1 / \tau_{\mathrm{nl}}(k) \sim k^{2 / 3}$, as illustrated in Figure 3. Here we depict an idealized inertial range behavior of $1 / \tau_{\mathrm{nl}}(k)$, extending from the outer scale to the Kolmogorov dissipation scale. Also shown are scale-dependent nonlinear rates computed from several types of simulation data, using the inertial range formula Equation (5). Included are data from simulations of 2D MHD, 3D Hall MHD, 3D PIC kinetic plasma (V. Roytershteyn et al. 2014, in preparation), and 2.5D hybrid Eulerian Vlasov (Servidio et al. 2012). In this selection of simulations the initial conditions and parameters have some similarities - rough equipartition of velocity and magnetic field fluctuations, minimal compressibility effects, equal viscosity and resistivities when possible, etc. However, the systems are not strictly controlled to be identical. For example, the mean field strengths vary (including no mean field for the 2D MHD case), and parameters such as the Hall parameter and mass ratio may differ. Data are taken at or near the time of peak meansquare current density. Further simulation details are given in Appendix B.

Note that in calculating $\tau_{\mathrm{nl}}(k)$ we employ twice the magnetic (omnidirectional) energy spectrum, i.e., $Z_{k}=\sqrt{2 k \mathcal{E}^{b}(k)}$, with the factor of two accounting for the approximately equal kinetic and magnetic contributions. Although this approach is not necessary with simulation data, it does facilitate later comparison with solar wind observations (Section 4), for which the cadence of the plasma (velocity) data is often much lower than that of the magnetic field measurements.

In presenting the simulation results, it is convenient to normalize wave numbers to the ion inertial length, $d_{\mathrm{i}}$. For the 


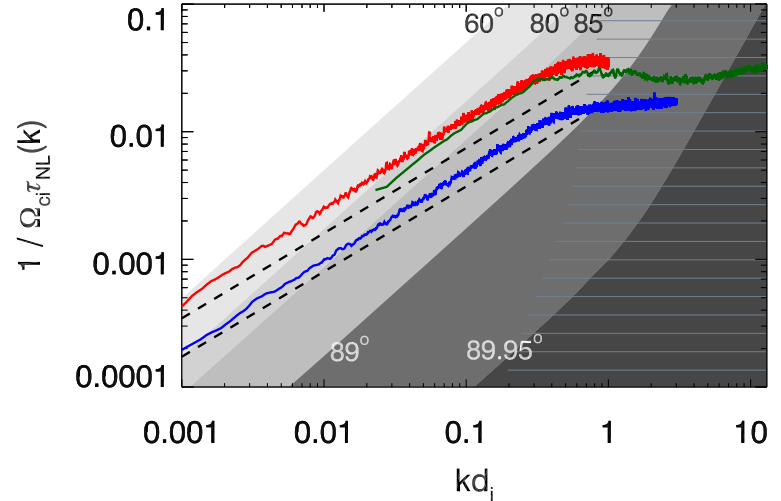

Figure 4. Nonlinear rates (reciprocal nonlinear times) for two fast solar wind intervals (red, green) and a slow solar wind interval (blue), compared to theoretical nonlinear rates for some typical solar wind parameters (upper dashed curve $Z^{2}=2000 \mathrm{~km}^{-2} \mathrm{~s}^{-2}$; lower dashed curve $Z^{2}=500 \mathrm{~km}^{-2} \mathrm{~s}^{-2}$ ); see text and Table 1. Also indicated are linear Vlasov theory determinations for characteristic wave frequencies (shaded regions), and damping rates (horizontal hatching). For kinetic scales ( $k d_{\mathrm{i}} \sim 1$ or greater), the observational nonlinear rates are comparable to the linear theory frequencies and rates associated with oblique angles. Shading delineates more oblique angle linear results, as in earlier figures.

(A color version of this figure is available in the online journal.)

kinetic cases $d_{\mathrm{i}}$ is intrinsic in the numerical formulation. For (one-fluid) MHD simulations this is not so, and there we have associated $d_{\mathrm{i}}$ with $\sqrt{43} \eta$, where $\eta$ is the Kolmogorov dissipation scale computed from the simulation and $43^{2} \approx 1836$ is the proton/electron mass ratio. This approach places the dissipation scale at the geometric mean of the ion and electron inertial scales. Consequently, all results in Figure 3 are presented using the normalization $\left[k d_{\mathrm{i}}, 1 /\left(\Omega_{\mathrm{ci}} \tau_{\mathrm{nl}}(k)\right)\right]$.

It is readily apparent that, in all cases, the nonlinear rates increase with $k$, until a steepened dissipation range is encountered, whether a well-defined inertial range is seen, or not. In the various simulations one always finds that the slope of the nonlinear rate passes through a region in which it is similar to the Kolmogorov value. As the dissipation range is approached, the spectral density of energy decreases more rapidly and the nonlinear rate levels off and usually decreases at very small scales. However in the upper inertial range-near $k d_{\mathrm{i}} \lesssim 1$ - the fastest nonlinear rates are entering the regime of kinetic rates since $1 / \Omega_{\mathrm{ci}} \tau_{\mathrm{nl}}(k) \approx 0.1-0.2$, i.e., not $\ll 1$. The nonlinear "fluid" timescales remain longer than the proton gyroperiod $\tau_{\mathrm{ci}}=2 \pi / \Omega_{\mathrm{ci}}$. However, the figure also shows that the nonlinear timescales in the crucial transition range between fluid and kinetic scales remain faster than essentially all frequencies of highly oblique $\theta>80^{\circ}$ linear waves, and faster than the associated damping rates of these waves. The nonlinear timescales are more than an order of magnitude faster than the wave frequencies of extremely oblique $\left(>89^{\circ}\right)$ fluctuations at $k d_{\mathrm{i}}=1$.

\section{LINEAR, NONLINEAR, AND SOLAR WIND TIMESCALES}

Linear and nonlinear processes are concurrent in a dynamic plasma and comparison of their characteristic timescales is a useful basis for discussing their relative effects. For example, when the nonlinear timescales are extremely long compared to the timescales computed for linear processes, then one expects those linear processes to occur without immediate modification. On the other hand, when nonlinear effects occur over a timescale comparable to, or shorter than, those of linear effects, one
Table 1

Some Parameters of the Solar Wind Intervals Used in Figure 4

\begin{tabular}{|c|c|c|c|c|}
\hline \multicolumn{2}{|l|}{ Quantity } & \multirow{2}{*}{$\begin{array}{c}\text { Wind } \\
384\end{array}$} & \multirow{2}{*}{$\frac{\text { STEREO }}{661}$} & \multirow{2}{*}{$\frac{\text { Cluster }}{666}$} \\
\hline$\left\langle\left|V_{s w}\right|\right\rangle$ & $\mathrm{km} \mathrm{s}^{-1}$ & & & \\
\hline$\left\langle\left|V_{A}\right|\right\rangle$ & $\mathrm{km} \mathrm{s}^{-1}$ & 70 & 65 & 60 \\
\hline$\langle|B|\rangle$ & $\mathrm{nT}$ & 7.4 & 3.7 & 4.5 \\
\hline$\left\langle\left|\delta V_{A}\right|^{2}\right\rangle$ & $\mathrm{km}^{-2} \mathrm{~s}^{-2}$ & 294 & 164 & 20 \\
\hline$\left\langle\left|\delta V_{s w}\right|^{2}\right\rangle$ & $\mathrm{km}^{-2} \mathrm{~s}^{-2}$ & 361 & 974 & 326 \\
\hline$\langle n\rangle$ & $\mathrm{cm}^{-3}$ & 5.3 & 1.5 & 2.6 \\
\hline$d_{\mathrm{i}}$ & $\mathrm{km}$ & 99 & 185 & 141 \\
\hline$\Omega_{\mathrm{ci}}$ & $\mathrm{rad} / \mathrm{s}$ & 0.71 & 0.36 & 0.43 \\
\hline$\beta_{\mathrm{p}}$ & & 0.3 & 0.6 & 2.5 \\
\hline$B$ resolution & $\mathrm{Hz}$ & 11 & 8 & 450 \\
\hline
\end{tabular}

must pause to reconsider how these processes interact with one another.

To affect such a comparison, Figure 4 presents a digest of frequencies from linear theory (shaded region), as in Figure 3, along with observationally determined nonlinear rates from three solar wind intervals. These observational estimates are based on $E^{b}(k)$, the reduced energy spectrum for the magnetic field, since the reduced spectrum for the velocity is often not available near the scales of interest here. To correct for this, we assume approximate equipartition of kinetic and magnetic energy at inertial range scales, i.e., we employ the slightly modified definition $1 / \tau_{\mathrm{nl}}(k)=k \sqrt{2 k E^{b}(k)}$. Here the omnidirectional spectrum $\mathcal{E}^{b}(k)$, usually used in theoretical work, has been replaced by the one-dimensional reduced spectrum $E^{b}(k)$, which is appropriate for spacecraft observations. The two spectra are related for special cases (Batchelor 1970).

We use $8 \mathrm{~Hz}$ magnetic field measurements from the IMPACT instrument (Acuña et al. 2008; Luhmann et al. 2008) and $1 \mathrm{~min}$ resolution proton plasma data from the PLASTIC instrument (Galvin et al. 2008) onboard the STEREO spacecraft in the ecliptic. A total of nine STEREO intervals are used, where all are in high-speed streams and contain no sector crossings. These intervals are identical to those used by Podesta (2009). Figure 4 shows nonlinear rate estimates (in red) from 2007 May 25 00:00 to 28 02:39 UT, which is typical of the stationary fast solar wind intervals used in this study. These are compared to data from slow solar wind intervals, where we use $11 \mathrm{~Hz}$ magnetic field measurements from the MFI instrument (Lepping et al. 1995) and $3 \mathrm{~s}$ resolution plasma data from the 3DP instrument (Lin et al. 1995) onboard the Wind spacecraft at 1 AU. Shown is a typical nonlinear rate estimate (in blue), from a slow solar wind stream in 2000 April 3 09:00 to 06 15:00 UT. The data in these intervals has been truncated from $k d_{\mathrm{i}}>1$ for STEREO and from $k d_{\mathrm{i}}>3$ for Wind, since noise becomes important at these scales and leads to an artificial flattening of the power spectral density. For completeness and in order to compare with kinetic simulations, we use high-frequency measurements of the magnetic field fluctuations from the search-coil (STAFF-SC; Cornilleau-Wehrlin et al. 1997) and flux-gate magnetometers (FGM; Balogh et al. 1997) onboard the Cluster spacecraft quartet to probe kinetic scales. We have chosen an interval where both STAFF-SC and FGM are operating in burst mode so that the smallest scales are accessible. Plasma data is obtained from the CIS HIA (Rème et al. 1997) instrument on Cluster 1. This is for a fast solar wind stream in 2007 January 30 00:10 to 01:20 UT. The estimates of the nonlinear rates are shown (in green) in Figure 4. The analysis is restricted to frequencies lower 
than $40 \mathrm{~Hz}$ to maintain a signal to noise ratio no less than $10 \mathrm{~dB}$ (Kiyani et al. 2009).

Also shown in Figure 4 are two theoretical curves, computed using $\Omega_{\mathrm{ci}}$ times Equation (5), and employing average solar wind-like parameters: $V_{A}=60 \mathrm{~km} \mathrm{~s}^{-1}$, correlation scale of $10^{6} \mathrm{~km}$, and $d_{\mathrm{i}}=100 \mathrm{~km}$ (i.e., density $\approx 5 \mathrm{~cm}^{-3}$ ). The curves differ due to the choice of squared fluctuation amplitude: $Z^{2}=500 \mathrm{~km}^{-2} \mathrm{~s}^{-2}$ and $Z^{2}=2000 \mathrm{~km}^{-2} \mathrm{~s}^{-2}$. For clarity we do not repeat the several simulation results in Figure 3.

The parameter space regions shaded in Figure 4 correspond to same prominent linear processes: wave oscillation frequencies, wave damping rates, and instability rates, as were depicted in Figure 3. These areas correspond to the discussion in Section 2 and the examples given in Figure 1. Evidently, the nonlinear rates are comparable to the linear ones near the onset of kinetic scales, $k d_{\mathrm{i}} \sim 1$ for sufficiently oblique spectral distributions of energy, and especially for $\theta>89^{\circ}$. Note that the Wind interval in particular (slow wind case) has a lower beta 0.3 for which the higher frequency dispersion relations should be shifted downward relative to the $\beta_{\mathrm{p}}=1$ shown here.

\section{DISCUSSION: NONLINEAR EFFECTS IN THE KINETIC REGIME}

It is apparent from Figure 4 that at length scales approaching $k d_{\mathrm{i}}=1$ from above, the rate of local-in-scale nonlinear processes overtakes and then exceeds a number of the kinetic plasma processes that have received significant attention regarding solar wind plasma dispersion and dissipation in the kinetic range. This is particularly true for highly oblique linear modes, which, for emphasis, are depicted using darker shading in Figures 1, 3, and 4. This effect may not have received sufficient consideration previously. Its implications, however, may differ subtly depending on which type of kinetic process is under consideration.

For linear waves, the influence of a fast nonlinear cascade is expected to modify the dynamical response of the system, i.e., the activity at a specified wave vector. Instead of being a simple oscillator with a characteristic frequency, one now has driving from larger scales, and damping by transfer of energy to smaller scales. So the problem becomes one of a stochastic nonlinear oscillator, which may exhibit behavior much different from simple harmonic motion. For example, random scrambling of a wave phase, even without energy change, causes a potentially dramatic frequency broadening (van Kampen 1992). Driving may also randomly change the energy content of the wave. Clearly, linear couplings will remain present and under some conditions linear wave properties may play an important role.

Turning to the damping rates, the situation becomes somewhat different. When the cascade rate at a particular scale becomes larger than the linear Vlasov damping rate $\gamma$, then the latter may become increasingly irrelevant. This may happen for example at a wave vector $\boldsymbol{k}$ when the nonlinear time is small enough that $\tau_{\mathrm{nl}}(\boldsymbol{k}) \ll 1 / \gamma(\boldsymbol{k})$. In this case for quasi-steady cascade conditions, most of the damping is due to dissipation not at $\boldsymbol{k}$, but in other (smaller scale) fluctuations. For example if the nonlinear cascade effect is much faster than linear damping of a particular KAW, then the energy may be transferred to much smaller scales and damped by other processes (including possibly damping of whistlers).

Finally, instability calculations may need be modified to account for nonlinear rates that exceed standard instability growth rates. Indeed it would seem that the problem of instability in a steady cascade becomes a perturbation about a driven dissipative steady-state, in contrast to one about an equilibrium. It is unclear whether the growth rates and other properties of usual mirror mode, AIC, and firehose instabilities (Gary 1993; Hellinger et al. 2006) will be changed substantially, and further detailed work on specific cases will be required to address this question. It is interesting to note that in the parameter space regions in which these instabilities are expected to act, there is also accumulating evidence of effects that might be attributable to enhanced turbulence (Bale et al. 2009; Osman et al. 2012, 2013; Servidio et al. 2014).

The above considerations suggest a natural measure of the degree to which the local ion kinetic physics is influenced by the nonlinear MHD-scale cascade. The relevant parameter appears to be

$$
\Phi\left(d_{\mathrm{i}}\right) \equiv \Omega_{\mathrm{ci}} \tau_{\mathrm{nl}}\left(k d_{\mathrm{i}}=1\right) .
$$

We have in mind a solar wind plasma with $\beta_{\mathrm{p}} \approx v_{\mathrm{th}}^{2} / V_{A}^{2} \sim 1$ for thermal speed $v_{\text {th }}$. For systems with more widely ranging $\beta_{\mathrm{p}}$, a more accurate indicator may be

$$
\Phi\left(\rho_{i}\right)=\Omega_{\mathrm{ci}} \tau_{\mathrm{nl}}\left(k \rho_{i}=1\right),
$$

where $\rho_{i}$ is the thermal proton gyroradius. Using the appropriate definition (which may vary according to specific cases), $\Phi<1$ indicates that the kinetic physics is strongly influenced by the MHD-scale cascade. However, since many relevant linear wave frequencies, damping rates, and instability growth rates in the solar wind are found at low frequencies $\lesssim \Omega_{\mathrm{ci}} / 10$, there may be significant nonlinear influences even when $\Phi\left(d_{\mathrm{i}}\right)$ is as large as 10 to 100

For the idealized inertial range, the above estimate of the normalized nonlinear timescale at $d_{\mathrm{i}}$ may be obtained using Equation (5):

$$
\Phi\left(d_{\mathrm{i}}\right) \equiv \Omega_{\mathrm{ci}} \tau_{\mathrm{nl}}\left(d_{\mathrm{i}}\right)=\frac{L \Omega_{\mathrm{ci}}}{Z} \frac{d_{\mathrm{i}}^{2 / 3}}{L^{2 / 3}}=\frac{V_{A}}{Z}\left(\frac{L}{d_{\mathrm{i}}}\right)^{1 / 3},
$$

a form that may be useful when $\beta_{\mathrm{p}} \sim 1$ and cross helicity $H_{c} \approx 0$. This can readily be generalized for other cases. The latter characterization should not be applied at scales smaller than $k d_{\mathrm{i}} \sim 1$, given that the form of $\tau_{\mathrm{nl}}(k)$ is likely different as discussed above. However a criterion for significance of nonlinear effects based on the more general form given in Equations (6) and (7) may remain valid even at $k d_{i}>1$.

We may note the relationship between $\Phi$ and a familiar measure of turbulence strength, the effective Reynolds number, which may be estimated as $R_{\text {eff }}=\left(L / d_{\mathrm{i}}\right)^{4 / 3}$. For nominal solar wind parameters, with $\beta_{\mathrm{p}}=1, L=10^{6} \mathrm{~km}, d_{\mathrm{i}}=100 \mathrm{~km}$, and $Z / V_{A}$ in the range $1 / 2$ to 1 , we find that $\Phi\left(d_{i}\right)=21-43$, indicating significant influence of nonlinear effects, especially in the parts of the spectrum that have wave vectors highly oblique to the mean magnetic field, as suggested in Figures 3 and 4.

The expectation that nonlinearities are strong in the solar wind as kinetic scales are approached is consistent with the detailed examples presented above, and motivates a careful look, possibly on a case-by-case basis, of the accuracy of wave damping and instability computed from linear theory for application to solar wind cascade, heating, and dissipation. It is noteworthy that the parameter $\Phi$ that we suggest here as an indicator of the expected influence of cascade on linear kinetic processes is also closely related to the controlling parameters identified in studies of test particle energization in various contexts, including acceleration to high energies, anisotropic generation of suprathermal particles, and plasma 
heating (Ambrosiano et al. 1988; Dmitruk et al. 2003, 2004; Chandran et al. 2010). Examination of physics-based parameters of the type given in Equation (8) may help to better understand how the intensity of turbulence influences the preferential absorption of energy into proton thermal energy as turbulence energy is increased, as recently reported based on PIC simulation (Wu et al. 2013).

As a final remark, we note that the present discussion has been based on a single nonlinear timescale that is characteristic of fluctuations at scale $1 / k$. Two types of complications enter immediately in any more detailed treatments of timescales. One is that the notion of locality in scale that we borrow from Kolmogorov theory applies to shells in wave vector space - that is, we associate a single nonlinear timescale to all wave vectors near a shell of wave number radius $k$. Thus, formulas for estimating $\tau_{\mathrm{nl}}(k)$ such as Equation (4) or (5) involve the total energy near the shell, that is the energy density integrated over a thin shell of radius $k$. This is a reasonable interpretation of locality even when the distribution of energy over the shell is anisotropic (see, e.g., Matthaeus et al. 2009). This necessarily involves averaging over regions (directions) on the shell that may have very different energy levels and spectral transfer properties. Nonlocal transfer and its associated timescales may be even more complex. We avoid all such theoretical complications here in an effort to elucidate the basic physical timescale competition between linear and nonlinear effects. Another underlying complication is that the characteristic timescale is only an average measure of the time variations at wave vector $\boldsymbol{k}$. In reality we know that time variations are broadband at each scale when nonlinear effects are strong. Stated another way, in turbulence the frequency $(\omega)$ spectra $P(\boldsymbol{k}, \omega)$ admit power over a broad range of frequency $\omega$ for a given wave vector $\boldsymbol{k}$, as seen for example in a variety of fluid, MHD, and plasma simulations (Dmitruk \& Matthaeus 2007, 2009; Parashar et al. 2010; Verscharen et al. 2012; TenBarge \& Howes 2012; TenBarge et al. 2013). The nonlinear timescale employed here is a standard estimate of the average effect due to local couplings for fluctuations in the inertial range. A more detailed treatment of the distribution of energy over timescales (or frequencies) would require examination of dynamical models in more detail than is warranted here. However, the present study may serve to motivate future more detailed studies of dynamical timescales in plasma turbulence.

This work is supported in part by NSF (AGS-1063439, AGS-1156094, SHINE), and by NASA (NNX09AG31G, NNX11AJ44G, NNX13AD72G, MMS-IDS NNX08A083G, MMS Theory and modeling Team, ISIS/Solar Probe Plus, and the Heliospheric Grand Challenges program), Turboplasmas project (Marie Curie FP7 PIRSES-2010-269297), POR Calabria FSE 2007/2013 and the UK STFC. Some simulations were done on NCAR Yellowstone supercomputers, and on Blue Waters (NSF ACI 1238993). Hybrid Vlasov-Maxwell simulations have been run within the European project PRACE Pra04-771.

\section{APPENDIX A}

\section{ALFVÉN CROSSING AND NONLINEAR TIMESCALES}

The Alfvén crossing time may be defined at the large scales as $\tau_{A}=L / V_{A}$, for energy-containing scale $L$ and large-scale Alfvén speed $V_{A}$ computed from the mean magnetic field $\boldsymbol{B}_{0}$ as $V_{A}=B_{0} / \sqrt{4 \pi \rho}$, for a given mass density $\rho$. By default we assume the large scales to be isotropic but the definition is readily generalized for imposed anisotropy. Whenever $V_{A}>Z \sim b$ for turbulence amplitude $Z$ and rms magnetic fluctuation $b$, the ordering $\tau_{A}<\tau_{\mathrm{nl}}$ holds. However, this timescale does not influence spectral transfer for strong turbulence, for reasons discussed below.

The wave vector dependent Alfvén time $\tau_{A}(\boldsymbol{k})=1 /\left|\boldsymbol{k} \cdot \boldsymbol{V}_{A}\right|$ is just the reciprocal of the MHD Alfvén wave frequency. For normal modes with substantial components $k_{\|}$parallel to $\boldsymbol{B}_{0}$, this timescale can be much shorter than the corresponding nonlinear time $\tau_{\mathrm{nl}}(\boldsymbol{k})$ and can in principle influence spectral transfer (Pouquet et al. 1976; Zhou et al. 2004). However, due to the usual dominant contribution of resonant triads to the nonlinear couplings (Shebalin et al. 1983; Grappin 1986; Oughton et al. 1994), the greatest contributions to nonlinear spectral transfer are independent of $\tau_{A}(\boldsymbol{k})$. In this regard it is crucial to recall that the wavelike couplings themselves make no contribution to spectral transfer. Rather the physics of Alfvénic wavelike couplings may be understood as mainly suppressing parallel spectral transfer, giving rise to spectral anisotropy (Shebalin et al. 1983; Carbone \& Veltri 1990; Oughton et al. 1994), but generally not having a major effect on the total rate at which spectral transfer occurs.

For highly anisotropic turbulence, the role of the Alfvén time may be of varying importance. For the most anisotropic case-purely two-dimensional turbulence-the largescale Alfvén time (computed in terms of the out-of-plane magnetic field) does not contribute at all. For low-frequency reduced MHD, the defining character of the dynamically important region of wave vector space is simply that $\tau_{\mathrm{nl}}(\boldsymbol{k}) \leqslant \tau_{A}(\boldsymbol{k})$ (Montgomery 1982). For critical balance turbulence, $\tau_{\mathrm{nl}}(\boldsymbol{k}) \sim$ $\tau_{A}(\boldsymbol{k})$, which is usually interpreted as $\tau_{\mathrm{nl}}(\boldsymbol{k}) \approx \tau_{A}(\boldsymbol{k})$ (Goldreich $\&$ Sridhar 1995). The Alfvén timescale is not an independent controlling factor for the rate of transfer in any of these cases. Consequently, in the analysis in the present paper, we focus exclusively on the nonlinear timescale for comparisons with the linear Vlasov timescales (where the Alfvén time again appears, but in connection with wave behavior).

\section{APPENDIX B}

\section{SIMULATION DETAILS}

For the 2D MHD case, the 2D incompressible MHD equations are solved in a $2 \pi$ periodic box using a Fourier spectral method. The simulation is a decaying run with initial kinetic and magnetic energies equal to 0.5 , and initial energy excited within a $k$-band of $[5,20]$. The resolution of the simulation is $16384 \times 16384$, with viscosity and resistivity $v=\eta=$ $2.0 \times 10^{-5}$ (Wan et al. 2013).

The 3D incompressible Hall MHD simulation is also a free decay run with initial kinetic and magnetic energies equal to 0.5 and an initially excited $k$-band of $[2,6]$. A Fourier spectral method is employed in a $2 \pi$-periodic cube with second-order Runge-Kutta time stepping. The resolution is 512 modes in each direction, with $1 / d_{\mathrm{i}}=25$, and $v=\eta=3.0 \times 10^{-3}$.

The 3D PIC simulation was performed using the general purpose PIC plasma simulation code VPIC (Bowers et al. 2008), which solves the relativistic Vlasov-Maxwell system of equations. The initial conditions correspond to uniform plasma with density $n_{0}$, Maxwellian-distributed ions and electrons of equal temperature $T_{0}$, a uniform magnetic field $B_{0} \hat{z}$, and have $\beta_{\mathrm{p}}=0.5$. The simulation domain is a cube of size $L \approx 41.9 d_{\mathrm{i}}$ with resolution of $2048^{3}$, such that the lowest allowed wavelength in each direction is $k_{\min } d_{\mathrm{i}}=0.15$. To reduce 
computational cost, the ion-to-electron mass ratio is taken to be $m_{i} / m_{e}=50$ in the simulation. The turbulence is seeded by imposing a perturbation of magnetic field initially, with the two lowest modes in each direction initialized (V. Roytershteyn et al. 2014, in preparation).

For the Vlasov simulation, the hybrid Vlasov-Maxwell equations are solved using an Eulerian algorithm, in a fivedimensional geometry (two dimensions in physical space and three in velocity space). The $2 \mathrm{D}$ plane is perpendicular to the mean field $\boldsymbol{B}_{0}$, and fluctuating vectors have three components, in general. The simulation is performed within a $\left(2 \pi \times 20 d_{\mathrm{i}}\right)^{2}$ box, with $512^{2}$ mesh points in space, and $51^{3}$ in the velocity space. The initial condition consists of a Maxwellian plasma perturbed by a $2 \mathrm{D}$ spectrum of Fourier modes, imposed for both the velocity and the magnetic fields. The plasma beta has been chosen equal to unity, and the level of fluctuations is $\delta b / B_{0}=0.66$. More details can be found in Servidio et al. (2012, 2014).

\section{REFERENCES}

Acuña, M. H., Curtis, D., Scheifele, J. L., et al. 2008, SSRv, 136, 203

Alexandrova, O., Carbone, V., Veltri, P., \& Sorriso-Valvo, L. 2008, ApJ, 674,1153

Alexandrova, O., Saur, J., Lacombe, C., et al. 2009, PhRvL, 103, 165003

Ambrosiano, J., Matthaeus, W. H., Goldstein, M. L., \& Plante, D. 1988, JGR, 93, 14383

Bale, S. D., Kasper, J. C., Howes, G. G., et al. 2009, PhRvL, 103, 211101

Bale, S. D., Kellogg, P. J., Mozer, F. S., Horbury, T. S., \& Reme, H. 2005, PhRvL, 94, 215002

Balogh, A., Dunlop, M. W., Cowley, S. W. H., et al. 1997, SSRv, 79, 65

Barnes, A. 1966, $\mathrm{PhFl}, 9,1483$

Barnes, A. 1968, ApJ, 154, 751

Barnes, A. 1969, ApJ, 155, 311

Barnes, A. 1979, in Solar System Plasma Physics, Vol. I, ed. E. N. Parker, C. F. Kennel, \& L. J. Lanzerotti (Amsterdam: North-Holland), 251

Batchelor, G. K. 1970, The Theory of Homogeneous Turbulence (Cambridge: Cambridge Univ. Press)

Belcher, J. W., \& Davis, L., Jr. 1971, JGR, 76, 3534

Biskamp, D., Schwarz, E., Zeiler, A., Celani, A., \& Drake, J. F. 1999, PhP1, 6, 751

Bowers, K. J., Albright, B. J., Yin, L., Bergen, B., \& Kwan, T. J. T. 2008, PhP1, 15,055703

Carbone, V., \& Veltri, P. 1990, GApFD, 52, 153

Chandran, B. D. G., Li, B., Rogers, B. N., Quataert, E., \& Germaschewski, K. 2010, ApJ, 720, 503

Chang, O., Gary, S. P., \& Wang, J. 2013, JGR, 118, 2824

Chen, S. Y., \& Kraichnan, R. H. 1989, PhFl A, 1, 2019

Cornilleau-Wehrlin, N., Chauveau, P., Louis, S., et al. 1997, SSRv, 79, 107

Dmitruk, P., \& Matthaeus, W. H. 2007, PhRvE, 76, 036305

Dmitruk, P., \& Matthaeus, W. H. 2009, PhPl, 16, 062304

Dmitruk, P., Matthaeus, W. H., \& Seenu, N. 2004, ApJ, 617, 667

Dmitruk, P., Matthaeus, W. H., Seenu, N., \& Brown, M. R. 2003, ApJ, 597, L81

Dobrowolny, M., Mangeney, A., \& Veltri, P. 1980, PhRvL, 45, 144

Frisch, U. 1995, Turbulence (Cambridge: Cambridge Univ. Press)

Galtier, S., \& Buchlin, É. 2007, ApJ, 656, 560

Galvin, A. B., Kistler, L. M., Popecki, M. A., et al. 2008, SSRv, 136, 437

Gary, S. P. 1993, Theory of Space Plasma Microinstabilities (New York: Cambridge Univ. Press)

Gary, S. P., \& Borovsky, J. E. 2004, JGR, 109, A06105

Gary, S. P., Saito, S., \& Li, H. 2008, GeoRL, 35, L02104

Goldreich, P., \& Sridhar, S. 1995, ApJ, 438, 763
Goldstein, M. L., Roberts, D. A., \& Matthaeus, W. H. 1995, ARA\&A, 33, 283 Grappin, R. 1986, PhFl, 29, 2433

Hellinger, P., Trávníček, P., Kasper, J. C., \& Lazarus, A. J. 2006, GeoRL, 33, L09101

Hollweg, J. V. 1999, JGR, 104, 14811

Howes, G. G., Cowley, S. C., Dorland, W., et al. 2006, ApJ, 651, 590

Howes, G. G., Cowley, S. C., Dorland, W., et al. 2008, JGR, 113, A05103

Isenberg, P. A., \& Vasquez, B. J. 2011, ApJ, 731, 88

Kiyani, K. H., Chapman, S. C., Khotyaintsev, Y. V., Dunlop, M. W., \& Sahraoui, F. 2009, PhRvL, 103, 075006

Kunz, M. W., Schekochihin, A. A., Cowley, S. C., Binney, J. J., \& Sanders, J. S. 2011, MNRAS, 410, 2446

Leamon, R. J., Matthaeus, W. H., Smith, C. W., et al. 2000, ApJ, 537, 1054

Leamon, R. J., Smith, C. W., Ness, N. F., \& Wong, H. K. 1999, JGR, 104, 22331

Lepping, R. P., Acuña, M. H., Burlaga, L. F., et al. 1995, SSRv, 71, 207

Lin, R. P., Anderson, K. A., Ashford, S., et al. 1995, SSRv, 71, 125

Luhmann, J. G., Curtis, D. W., Schroeder, P., et al. 2008, SSRv, 136, 117

Lysak, R. L., \& Lotko, W. 1996, JGR, 101, 5085

MacBride, B. T., Smith, C. W., \& Forman, M. A. 2008, ApJ, 679, 1644

Marino, R., Sorriso-Valvo, L., Carbone, V., et al. 2008, ApJ, 677, L71

Maruca, B. A., Kasper, J. C., \& Bale, S. D. 2011, PhRvL, 107, 201101

Matteini, L., Landi, S., Hellinger, P., et al. 2007, GeoRL, 34, L20105

Matthaeus, W. H., Goldstein, M. L., \& Roberts, D. A. 1990, JGR, 95, 20673

Matthaeus, W. H., Minnie, J., Breech, B., et al. 2004, GeoRL, 31, L12803

Matthaeus, W. H., Oughton, S., \& Zhou, Y. 2009, PhRvE, 79, 035401

Matthaeus, W. H., \& Velli, M. 2011, SSRv, 160, 145

Montgomery, D. C. 1982, PhyS, T2/1, 83

Osman, K. T., \& Horbury, T. S. 2007, ApJL, 654, L103

Osman, K. T., Matthaeus, W. H., Hnat, B., \& Chapman, S. C. 2012, PhRvL, 108,261103

Osman, K. T., Matthaeus, W. H., Kiyani, K. H., Hnat, B., \& Chapman, S. C. 2013, PhRvL, 111, 201101

Oughton, S., Priest, E. R., \& Matthaeus, W. H. 1994, JFM, 280, 95

Parashar, T. N., Servidio, S., Breech, B., Shay, M. A., \& Matthaeus, W. H. 2010, PhPl, 17, 102304

Podesta, J. J. 2009, ApJ, 698, 986

Pouquet, A., Frisch, U., \& Léorat, J. 1976, JFM, 77, 321

Rème, H., Bosqued, J. M., Sauvaud, J. A., et al. 1997, SSRv, 79, 303

Riquelme, M. A., Quataert, E., Sharma, P., \& Spitkovsky, A. 2012, ApJ, 755,50

Roberts, D. A., Goldstein, M. L., Klein, L. W., \& Matthaeus, W. H. 1987, JGR, 92, 12023

Roberts, O. W., Li, X., \& Li, B. 2013, ApJ, 769, 58

Sahraoui, F., Belmont, G., \& Goldstein, M. L. 2012, ApJ, 748, 100

Sahraoui, F., Goldstein, M. L., Belmont, G., Canu, P., \& Rezeau, L. 2010, PhRvL, 105, 131101

Schekochihin, A. A., Cowley, S. C., Rincon, F., \& Rosin, M. S. 2010, MNRAS, 405, 291

Servidio, S., Carbone, V., Dmitruk, P., \& Matthaeus, W. H. 2011, Europhys. Lett., 96, 55003

Servidio, S., Osman, K. T., Valentini, F., et al. 2014, ApJL, 781, L27

Servidio, S., Valentini, F., Califano, F., \& Veltri, P. 2012, PhRvL, 108, 045001

Sharma, P., Quataert, E., Hammett, G., \& Stone, J. M. 2007, ApJ, 667, 714

Shebalin, J. V., Matthaeus, W. H., \& Montgomery, D. 1983, JPIPh, 29, 525

Sorriso-Valvo, L., Marino, R., Carbone, V., et al. 2007, PhRvL, 99, 115001

TenBarge, J. M., \& Howes, G. G. 2012, PhPl, 19, 055901

TenBarge, J. M., Howes, G. G., \& Dorland, W. 2013, ApJ, 774, 139

Tu, C.-Y., \& Marsch, E. 1995, SSRv, 73, 1

van Kampen, N. G. 1992, Stochastic Processes in Physics and Chemistry (3rd ed.; Amsterdam: Elsevier)

Verscharen, D., Marsch, E., Motschmann, U., \& Müller, J. 2012, PhPl, 19, 022305

Wan, M., Matthaeus, W. H., Servidio, S., \& Oughton, S. 2013, PhP1, 20, 042307

Wu, P., Wan, M., Matthaeus, W. H., Shay, M. A., \& Swisdak, M. 2013, PhRvL, 111,121105

Zhou, Y., Matthaeus, W. H., \& Dmitruk, P. 2004, RvMP, 76, 1015 